\title{
The flashy disappearance of a star falling behind the horizon of a black hole
}

\author{
A. Čadež and A. Gomboc \\ University of Ljubljana, Department of Physics, Jadranska 19, 61000 Ljubljana, Slovenia \\ E-mail: andrej.cadez@uni-lj.si, andreja@samson.fiz.uni-lj.si
}

Received December 6, 1995; accepted March 7, 1996

\begin{abstract}
We present calculations and images of the appearance of a point-like star while heading toward and disappearing behind the horizon of a Schwarzschild black hole. A complete review of equations of motion and their solutions is given in Sect. 2, and Sect. 3 describes the properties of the star as the light source. The results of numerical simulations are shown in 26 figures and some phenomena responsible for their appearance are discussed in Sect. 4 . It is possible that such phenomena are relevant for the description of certain activities related to galactic black holes and also for the description of $\gamma$-ray burstars.
\end{abstract}

Key words: black holes physics — gravitational lensing — gamma rays: bursts

\section{Introduction}

It has been often said that a particle falling into a black hole would never be seen by an outside observer to cross the horizon, because it takes an infinite amount of coordinate time for the particle to reach the horizon. As important as this illustration might be for the description of geometrical properties of the black-hole space-time, it tells little to the astrophysicist what he might expect to see, should he really have a chance to observe such a phenomenon. It seems to us that the phenomenon of a black hole devouring a radiating particle can most directly be described by constructing a numerical simulation of such an event and presenting the result as a video representation. Having done this, we can understand the mechanisms responsible for different phases of the appearance of the approach and final disappearance of a particle while approaching the black hole. In the first phase the important effect is the acceleration of the particle to high speed in the vicinity of the black hole. Due to aberration of light and the Doppler shift the particle appears brighter and bluer to observers in the direction of the particle's motion and dimmer and redder to those behind. As the particle approaches the black hole, the gravitational lensing also becomes important in focusing the light for a particular set of observers. By the time the particle reaches the vicinity of the radius $r=3 M$ (in Schwarzschild coordinates), both the aberration and gravitational lensing work together to make the infalling particle appear considerably brighter to

Send offprint requests to: A. Čadež those observers in front of the moving particle. However, after the radius $r=3 \mathrm{M}$ is passed, the percentage of photons emitted by the infalling particle and caught by the black hole starts increasing dramatically, so that only a rapidly decreasing number of photons, moving in the opposite direction than the infalling particle, reach the far observer as ever dimmer and redder light. Thus, the disappearance of the particle from the eyes of a far observer is a rather characteristic process. It takes a few hundred $G M / c^{3}$ units for the star to brighten up and blueshifting its spectrum (it may do this in short bursts), before it reaches the maximum. After this, the stellar luminosity abruptly decays into oblivion in only a few $G M / c^{3}$ units. The final disappearance of the particle from the eyes of a far observer is exponential with the decay time of only $G M / c^{3} \sqrt{3}$ (Misner et al. 1973).

The rapid luminosity and redshift change are two characteristic features of the disappearance of a star behind the horizon of a black hole. No other process can produce as fast a luminosity and redshift change in a star (Misner et al. 1973). In this respect it is interesting to note the shortness of the often abrupt and definitive end of a burstar. Some burst ends (CGRO) roughly fit a description of falling behind a horizon whose radius $\left(G M / c^{2}\right)$ corresponds to less then a few ten solar masses. Such an interpretation of the end of the burst is in accordance with models proposing that burstars are collapsing binary neutron stars (Piran 1995). Falling behind the horizon of a galactic black hole could also be a process, which would display the characteristic sharp decline of luminosity 
accompanied by a rapidly changing redshift. Such a process has been considered by Luminet et al. (Luminet \& Barbuy 1990; Luminet \& Carter 1986; Luminet \& Marck 1985; Luminet \& Pichon 1989a; Luminet \& Pichon 1989b) and Rees (Rees 1988; Rees 1990). We note that in this case the shortest characteristic decay time revealing the final approach to the black hole horizon is a few hours up to a day, and the flaring up process which we described above as due to aberration of light and gravitational lensing, is about a hundred times longer. Again, one might be wondering if the rapid luminosity changes of AGN's might not tell us about such phenomena.

It would be difficult to treat all the scenarios in realistic detail. Therefore, in our first attempt we consider an isotropic point-like source falling down a Schwarzschild black hole. This solution is similar to a Green's function for a more general astrophysical problem which should include some degree of tidal disruption of the star after passing the Roche radius (Carter \& Luminet 1985; Luminet \& Marck 1985). However, if a lower main sequence star is falling into a large galactic black hole $\left(M>\approx 10^{8} M_{\odot}\right)$ the problem is almost as simple as our calculation, since the Roche radius is behind the horizon and thus the star would only be marginally deformed. The modest squeezing of the star on the way toward the black hole might be just sufficient to start a nuclear detonation, which makes the star brighten enough to be observable.

\section{Equations of motion and their solutions}

The equations of motion of a particle or a photon in a gravitational field of a Schwarzschild and a more complicated Kerr black hole have been extensively treated in the literature (Bardeen 1972; Misner et al. 1973; Rauch \& Blandford 1994; Weinberg 1972) using different formalisms.

In the following we derive the solutions of the equations of motion in Hamiltonian formalism using the complete set of constants of motion. We justify some repetition of already published results by the necessity of having an efficient and numerically stable system of equations describing the motion of light in Schwarzschild space-time in order to be able to fully implement the ray-tracing technique.

The equations of motion of a particle or a photon in a gravitational field are well known to be derivable from the Lagrangian:

$$
L=\sqrt{-g_{\mu \nu} \dot{x}^{\mu} \dot{x}^{\nu}}
$$

Here $x^{\mu}(\mu=0, \ldots 3)$ are generalised coordinates describing the space-time position of the particle as a function of its proper time $\tau, g_{\mu \nu}$ are the components of the metric tensor of the space-time under consideration and the dot denotes differentiation with respect to the proper time $\tau$. The Lagrangian itself is a constant of motion having the value $c$ for particles following timelike geodesics and 0 for photons. Because of this constancy, any (smooth) function of the Lagrangian (1) is an equivalent generator of equations of motion. Thus, we will use the form:

$$
L^{\prime}=\frac{1}{2} g_{\mu \nu} \dot{x}^{\mu} \dot{x}^{\nu}
$$

and switch to the Hamiltonian formalism. To his end we calculate the canonical momenta:

$$
p_{\mu}=\frac{\partial L^{\prime}}{\partial \dot{x}^{\mu}}
$$

and the Hamiltonian:

$$
H=p_{\mu} \dot{x}^{\mu}-L^{\prime}=\frac{1}{2} g^{\mu \nu} p_{\mu} p_{\nu}
$$

where $g^{\mu \nu}$ are the components of the matrix, which is the inverse of $g_{\mu \nu}$. The Hamiltonian (4) is of course also a constant of motion with the value $-\frac{1}{2} c^{2}$ for particles travelling on timelike geodesics and 0 for photons (From now on we will use the units such that $c=G=1$ ). In the photon case the scale of the parameter $\tau$ is arbitrary and we choose it so that $p_{\mu}$ are the components of the 4-momentum. In the Schwarzschild space-time the Hamiltonian is explicitly:

$$
H=\frac{1}{2}\left[-\frac{1}{1-\frac{2 M}{r}} p_{0}^{2}+\left(1-\frac{2 M}{r}\right) p_{r}^{2}+\frac{1}{r^{2}}\left(p_{\theta}^{2}+\frac{1}{\sin ^{2} \theta} p_{\varphi}^{2}\right)\right]
$$

We note that $x^{0}$ and $\varphi$ are cyclic coordinates, so that their conjugate momenta $p_{0}$ and $p_{\varphi}$ are constants of motion. These observations show, that the Hamiltonian is of exactly the same form as the nonrelativistic Hamiltonian describing the motion of a particle in a central potential. Therefore, the full angular momentum is also a constant of motion. Indeed, by computing the Poisson brackets of the angular momentum components:

$l_{z}=p_{\varphi} \quad$ and $\quad l_{ \pm}=l_{x} \pm i l_{y}=\left(\cot \theta p_{\varphi} \mp i p_{\theta}\right) \mathrm{e}^{ \pm i \varphi}$

with the Hamiltonian (5), it is not difficult to show that they vanish. The square of the angular momentum:

$$
l^{2}=l_{x}^{2}+l_{y}^{2}+l_{z}^{2}=l_{+} l_{-}+l_{z}^{2}=p_{\theta}^{2}+\frac{1}{\sin ^{2} \theta} p_{\varphi}^{2}
$$

is of course also a constant of motion. Inserting (7) into the Hamiltonian (5) we obtain an equation for $p_{r}$ as a function of $r$. Using the Hamilton equation

$$
\dot{r}=\frac{\partial H}{\partial p_{r}}=\left(1-\frac{2 M}{r}\right) p_{r}
$$

one expresses $p_{r}$ as a function of $\dot{r}$ and $r$ and thus obtains a first order differential equation for $r$ as a function of $\tau$ :

$$
\dot{r}^{2}=2 H\left(1-\frac{2 M}{r}\right)+p_{0}^{2}-\frac{l^{2}}{r^{2}}\left(1-\frac{2 M}{r}\right)
$$

This equation may be solved by an integration. 


\subsection{The orbit equation}

At this point it is useful to look for the orbit equation. We first seek the canonical variable, call it $\lambda$, which is conjugate to the component of the angular momentum $l=\boldsymbol{l} \cdot \hat{n}$, where $\hat{n}$ in the direction of the angular momentum itself. In the notation usual to spherical astronomy the directions of angular momentum components are written as:

$$
\left\{n_{x}, n_{y}, n_{z}\right\}=\{\sin \varepsilon \sin \Omega,-\sin \varepsilon \cos \Omega, \cos \varepsilon\}
$$

so that the angular momentum $l$ becomes:

$l=[\sin \varepsilon \cot \theta \sin (\varphi-\Omega)+\cos \varepsilon] p_{\varphi}+\sin \varepsilon \cos (\varphi-\Omega) p_{\theta}$

The value of the Poisson bracket between conjugate variables must be 1 , so that we must have:

$$
[\lambda, l]=1
$$

and also:

$$
\left[\varphi, p_{\varphi}\right]=1 \quad \text { and } \quad\left[\theta, p_{\theta}\right]=1
$$

Using the Poisson bracket algebra it is easy to show that if:

$$
\cos (\lambda+\omega)=\cos (\varphi-\Omega) \sin \theta \text { and } \sin (\lambda+\omega)=-\frac{\cos \theta}{\sin \varepsilon}
$$

are satisfied, then $\lambda$ obeys the Poisson bracket relation (12) and $\theta$ and $\varphi$ can be expressed with $\lambda$ as follows:

$$
\begin{aligned}
\cos \theta & =-\sin \varepsilon \sin (\lambda+\omega) \\
\tan (\varphi-\Omega) & =\cos \varepsilon \tan (\lambda+\omega)
\end{aligned}
$$

Here $\omega$ is an integration constant which is usually called the longitude of the periastron.

The orbit equation is the relation between $\lambda$ and $r$. It can be obtained by using the Hamilton equation:

$$
\dot{\lambda}=[\lambda, H]=\left[\lambda, \frac{l^{2}}{2 r^{2}}\right]=\frac{l}{r^{2}}
$$

and the Eq. (9). Using (17) one obtains $\dot{r}=\frac{\mathrm{d} r}{\mathrm{~d} \lambda} \dot{\lambda}=\frac{\mathrm{d}}{\mathrm{d} \lambda}\left(\frac{l}{r}\right)$, and upon inserting this into (9) we obtain:

$$
\frac{\mathrm{d}}{\mathrm{d} \lambda}\left(\frac{l}{r}\right)= \pm \sqrt{2 H\left(1-\frac{2 M}{r}\right)+p_{0}^{2}-\frac{l^{2}}{r^{2}}\left(1-\frac{2 M}{r}\right)}
$$

Introducing a new variable:

$$
u=\frac{2 M}{r}
$$

Eq. (18) may be cast into the form:

$$
\frac{\mathrm{d} u}{\mathrm{~d} \lambda}= \pm \sqrt{a^{2}+b(1-u)-u^{2}(1-u)}
$$

where:

$$
a=2 M \frac{p_{0}}{l} \quad \text { and } \quad b=2 H\left(\frac{2 M}{l}\right)^{2}
$$

The radical under the square root of Eq. (20) is a third degree polynomial, so that the equation is generally solvable in terms of elliptic functions. It is worth noting that we are mostly interested in photon orbits for which $b=0$ and the radical of Eq. (20) assumes a more symmetric form for which the said radical can be factorized as follows:

a) if $a<\frac{2}{3 \sqrt{3}}$, then we define:

$$
\psi=2 \arcsin \frac{1}{2} 3 \sqrt{3} a
$$

and

$$
a^{2}-u^{2}(1-u)=\left(u-u_{1}\right)\left(u-u_{2}\right)\left(u-u_{3}\right)
$$

where

$$
\left(\begin{array}{c}
u_{1}=\frac{1}{3}\left(1+2 \cos \frac{\psi}{3}\right) \\
u_{2}=\frac{1}{3}\left(1-2 \cos \frac{\psi+\pi}{3}\right) \\
u_{3}=\frac{1}{3}\left(1-2 \cos \frac{\psi-\pi}{3}\right)
\end{array}\right)
$$

Note that $u_{1}>u_{2}>u_{3}$ and $u_{3}<0$ and $u_{2}>0$. Thus, photon orbits are possible for $\frac{1}{u_{2}} \leq \frac{r}{2 M} \leq \infty$ and $0 \leq \frac{r}{2 M} \leq \frac{1}{u_{1}}$. For the first type of orbits $u_{2}$ determines the periastron $\left(r_{\text {per }}=\frac{2 M}{u_{2}}\right)$, while orbits of the second type (the negative energy orbits) never reach infinity and are not interesting for the present consideration.

b) if $a>\frac{2}{3 \sqrt{3}}$, then we define:

$$
D=\left[\frac{27}{2} a^{2}-1-\sqrt{\left(\frac{27}{2} a^{2}-1\right)^{2}-1}\right]^{\frac{1}{3}}
$$

and

$$
\begin{aligned}
& a^{2}-u^{2}(1-u)= \\
& =\left\{\left[u-\frac{1}{3}\left(1+\frac{1}{2}\left(D+\frac{1}{D}\right)\right)\right]^{2}+\frac{1}{36}\left(\frac{1}{D}-D\right)^{2}\right\}\left(u-u_{1}\right)
\end{aligned}
$$

where

$$
u_{1}=\frac{1}{3}\left(1-\frac{1}{D}-D\right)
$$

Note that $u_{1}$ - the only real zero of the polynomial is always negative, so that the corresponding photons always end (in the past or in the future) inside the black hole.

The solutions of orbit Eq. (18) can now be found through some manipulations and with the help of mathematical tables (Gradshteyn \& Ryzhik 1965) as:

in case $\mathbf{a})$ if $\lambda$ is reckoned from the periastron:

$$
\begin{array}{r}
\lambda(r)= \pm 2 \sqrt{\frac{\sin \frac{\pi}{3}}{\sin \frac{\psi+\pi}{3}}}\left\{F\left(\frac{\pi}{2}, \sqrt{\frac{\sin \frac{\psi}{3}}{\sin \frac{\psi+\pi}{3}}}\right)-\right. \\
-F\left(\frac{\pi}{2}-\frac{1}{2} \arccos \left(\frac{\frac{2 M}{r}-\frac{1}{3}\left(1-\cos \frac{\psi}{3}\right)}{\sin \frac{\psi}{3}} \sqrt{3}\right), \sqrt{\left.\frac{\sin \frac{\psi}{3}}{\sin \frac{\psi+\pi}{3}}\right)}\right\}
\end{array}
$$


where the sign is + after periastron passage and - before.

in case $\mathbf{b}$ ) there is no periastron, so that $\lambda$ is reckoned from the initial position (i):

$$
\begin{aligned}
& \lambda(r)=\left(\frac{48}{\frac{1}{D^{2}}+1+D^{2}}\right)^{\frac{1}{4}} \\
& \left\{F\left(\arcsin \sqrt{\frac{1+\tan ^{2} \frac{\mu}{2}}{1+\tan \frac{\mu}{2} \mathrm{e}^{\zeta(r)}}}, \cos \frac{\mu}{2}\right)-\right. \\
& \left.-F\left(\arcsin \sqrt{\frac{1+\tan ^{2} \frac{\mu}{2}}{1+\tan \frac{\mu}{2} \mathrm{e}^{\zeta\left(r_{i}\right)}}}, \cos \frac{\mu}{2}\right)\right\}
\end{aligned}
$$

where

$$
\tan \mu=\frac{1}{\sqrt{3}} \frac{1-D^{2}}{1+D^{2}}
$$

and

$$
\zeta(r)=\operatorname{arcsinh} \frac{\frac{2 M}{r}-\frac{1}{3}\left[1+\left(\frac{1}{D}+D\right) \cos \frac{\pi}{3}\right]}{\frac{1}{3}\left(\frac{1}{D}-D\right) \sin \frac{\pi}{3}}
$$

and $r_{i}$ is the radial coordinate of the initial position.

\subsection{Constants of motion, initial and final data}

We are interested in calculating orbits of photons emanating from a star which is moving towards a black hole. Therefore, we take the orbital plane as the basic plane $\left(\theta=\frac{\pi}{2}\right)$ of the system of coordinates. A point on this plane, where the star is found (usually) in infinite past, defines the position, where the coordinate $\varphi$ takes the value $\pi$. The emission of any photon is thus characterised by initial coordinates $t_{i}, r_{i}, \varphi_{i}$ and $\theta_{i}=\frac{\pi}{2}$ and by the initial direction of photon's motion characterised by $\dot{r}_{i}, \dot{\theta}_{i}$ and $\dot{\varphi}_{i}$. From initial data we first calculate the components of angular momentum. Noting that $\theta_{i}=\frac{\pi}{2}$ one finds from (6)

$$
l_{z}=r_{i}^{2} \dot{\varphi}_{i} \quad \text { and } \quad l_{ \pm}=\mp i r_{i}^{2} \dot{\theta}_{i} \mathrm{e}^{ \pm i \varphi_{i}}
$$

Comparing this with (10), we find:

$$
\begin{aligned}
l & =r_{i}^{2} \sqrt{\dot{\theta}_{i}^{2}+\sin ^{2} \theta_{i} \dot{\varphi}_{i}^{2}} \\
\Omega & =\varphi_{i} \\
\tan \varepsilon & =\frac{\dot{\theta}_{i}}{\dot{\varphi}_{i}}
\end{aligned}
$$

Furthermore (15) gives (noting that $\theta_{i}=\frac{\pi}{2}$ ):

$$
\lambda_{i}+\omega=n \pi
$$

and (16) confirms (34). The longitude of the periastron is a useful concept only if the periastron exists, thus with regard to (36) we must distinguish between the case a) and $\mathbf{b}$ ) of the previous section. Case $\mathbf{a}$ ): the periastron exists and:

i) if $\dot{r}_{i}<0$, then the photon is approaching the black hole and will reach the periastron after $\lambda$ has increased by $\omega$, so that:

$$
\omega=-\lambda\left(r_{i}\right)
$$

ii) if $\dot{r}_{i}>0$ the photon is moving away from the black hole and:

$$
\omega=\pi-\lambda\left(r_{i}\right)
$$

Case $\mathbf{b}$ ): there is no periastron and only the photons with $\dot{r}_{i}>0$ will reach the observer at infinity, those with $\dot{r}_{i}<0$ will be swallowed by the black hole and are of no interest to us. For the photons reaching the far observer $r$ is a monotonously increasing function of time, so that $\lambda$ may be reckoned from the initial position by putting $\omega=0$.

Having found all the constants of motion, one can easily calculate the celestial coordinates $\theta_{\mathrm{f}}$ and $\varphi_{\mathrm{f}}$, where the photon reaches the celestial sphere at $r_{\mathrm{f}} \gg M$. Finally, we must find the time of flight of the photon from the point of emission to the point of detection in order to know in which image the photon will be detected. To this end we note that:

$$
\dot{t}=\frac{\partial H}{\partial p_{0}}=-\frac{p_{0}}{1-\frac{2 M}{r}}
$$

Combining this equation with (9), we get the following equation relating the radial coordinate $(r)$ and the coordinate time $(t)$ :

$$
\frac{\mathrm{d} r}{\mathrm{~d} t}= \pm \frac{1}{a}\left(1-\frac{2 M}{r}\right) \sqrt{a^{2}-\left(\frac{2 M}{r}\right)^{2}\left(1-\frac{2 M}{r}\right)}
$$

We solve (40) numerically by integrating it from $r_{i}$ to $r_{\mathrm{f}}$ to obtain $t_{\mathrm{f}}-t_{i}$. In doing this numerical integration one must again distinguish cases $\mathbf{a}$ ) and $\mathbf{b}$ ) from the previous section.

In case a)i) where the photon is first heading toward the periastron it is convenient to start the integration at the periastron and calculating the time lapse $t_{\mathrm{f}}-t_{i}$ as the sum of the two time lapses, one from the periastron to $r_{i}$ and the other from periastron to $r_{\mathrm{f}}$. The numerical integration can be done quite efficiently if one starts at the periastron by expanding $r(t)$ into a Taylor series, of which we write the first few terms:

$$
r(t)=2 M / u_{2}+\frac{1}{2} r^{\prime \prime}\left(t-t_{\mathrm{per}}\right)^{2}+\frac{1}{24} r^{i v}\left(t-t_{\mathrm{per}}\right)^{4}
$$

where

$$
\begin{aligned}
r^{\prime \prime} & =\frac{2 M}{(2 M a)^{2}}\left(1-u_{2}\right)^{2} u_{2}^{3}\left(1-\frac{3}{2} u_{2}\right) \\
r^{i v} & =-\frac{u_{2}^{4}}{(2 M a)^{2}}\left(1-u_{2}\right)\left(4 u_{2}-3\right)\left(3 u_{2}-1\right) r^{\prime \prime}
\end{aligned}
$$

Note, however, that if $a$ approaches $\frac{2}{3 \sqrt{3}}$ from below, then $u_{2}$ goes to $\frac{2}{3}$ and all the derivatives $r^{\prime \prime}, r^{i v}, \ldots$ go to zero. In this case it is convenient to introduce a new coordinate

$$
z=r+3 M \log \left(\frac{r}{3 M}-1\right)
$$


which satisfies:

$$
\frac{\mathrm{d} z}{\mathrm{~d} t}=\frac{1}{1-\frac{3 M}{r}} \frac{\mathrm{d} r}{\mathrm{~d} t}
$$

so that in the vicinity of the periastron the derivatives of $z$ with respect to the coordinate time $t$ are finite and nonvanishing. Thus, $z$ is expanded in a Taylor series in the vicinity of the periastron as:

$$
\begin{aligned}
z(t) & =2 M / u_{2}+3 M \log \left(\frac{2}{3 u_{2}}-1\right)+\frac{1}{2} z^{\prime \prime}\left(t-t_{\mathrm{per}}\right)^{2}+ \\
& +\frac{1}{24} z^{i v}\left(t-t_{\mathrm{per}}\right)^{4}
\end{aligned}
$$

where

$$
\begin{aligned}
z^{\prime \prime} & =\frac{2 M}{(2 M a)^{2}}\left(1-u_{2}\right)^{2} u_{2}^{3} \\
z^{i v} & =-\frac{u_{2}^{4}}{(2 M a)^{2}}\left(1-u_{2}\right)\left(4 u_{2}-3\right)\left(3 u_{2}-1\right) z^{\prime \prime}- \\
& -\frac{9 u_{2}^{2}}{4 M}\left(z^{\prime \prime}\right)^{2}
\end{aligned}
$$

We calculate $z$ for a suitable time $t$ such that the expansion (46) is still valid and invert (44) to get the starting coordinate $r(t)$ for solving the Eq. (40). The fourth order Runge-Kutta integration (with adjusted time step) gives sufficient accuracy (a few thousands) after only a few ten steps.

In case a)ii) the photon is moving away from the periastron and the numerical integration of (40) usually gives a sufficiently fast and accurate result. Only if the photon is emitted very close to its periastron the intermediate variable $z$ becomes helpful.

Case $\mathbf{b}$ ): in this case the photons have no periastron and the time of flight for those reaching the faraway observer may be obtained directly by integrating the Eq. (40). This equation again is well behaved for $a>\frac{2}{3 \sqrt{3}}$ but becomes problematic as $a \rightarrow \frac{2}{3 \sqrt{3}}$ and if the photon is emitted at $r_{i}<3 M$. Such photon trajectories wind around the sphere $r=3 M$ before unwinding into straight lines stretching to infinity. They are very few and trickle out as last messengers, so they turn out not to be very important for the happening. Their time of flight is best calculated in three steps. First one calculates the time of flight from $r_{i}$ to $r_{-}=3 M-\epsilon$ by numerically integrating the Eq. (40), where $\epsilon$ is a small distance for example $0.1 \times M$. Next one calculates the winding angle $\Delta \lambda_{w}$ of the trajectory between $r_{-}$and $r_{+}=3 M+\epsilon$ according to Eqs. (29), (30) and (31). Since the trajectory is winding very close to $r=3 M$, the time it takes to wind $\left(\Delta t_{w}\right)$ can be calculated with sufficient precision using (17) and (39) and taking $r=3 M$. Thus one obtains:

$$
\Delta t_{w}=\frac{27 M a}{2} \Delta \lambda_{w}
$$

The remaining time of flight between $r_{+}$and $r_{\mathrm{f}}$ is calculated by numerically integrating the Eq. (40).

\section{Modeling the emission source}

As described above, the emission source is a star falling into the black hole. The star is considered as a point particle following a time-like geodesic $X^{\mu}(\tau)(=\{t, R, \Theta, \Phi\})$, which obeys the equations of motion following from the Hamiltonian (5), which takes the value $-\frac{1}{2} c^{2}$. Since the stellar orbit is the basic plane of the system of coordinates, the only nonzero component of the stellar angular momentum is the $z$ component $L=L_{z}=P_{\varphi}\left(L_{+}=L_{-}=0\right)$. The stellar orbit is thus described by two nontrivial constants of motion $L$ and $P_{0}$.

i) If $P_{0}>1$, then the stellar orbit has one end (the infinite past) at spatial infinity, while the other end (the future) may be either at spatial infinity (for unbound orbits) or behind the horizon (for plunging orbits). The future end of the orbit depends on the value of the angular momentum; if $L>L_{\text {crit }}$, then the orbit is unbound, otherwise it is a plunging orbit. $L_{\text {crit }}$ depends on the value of $P_{0}$ (for example if $P_{0}=1$, then $L_{\text {crit }} / M=4$ )

ii) If $P_{0}<1$, then the orbit can not extend to infinity and is either a Keplerian bound orbit for sufficiently large angular momentum, or a plunging orbit for small angular momentum.

As the star is moving along the geodesic, it is radiating light (photons) uniformly in its own rest-frame. The luminosity per unit solid angle and per unit frequency is described by:

$$
I_{\nu}^{\prime}\left(\hat{n}^{\prime}\right)=\frac{L_{0}}{4 \pi} \delta\left(\nu-\nu_{0}\right)
$$

Here $L_{0}$ is the total luminosity of the "monochromatic" star radiating at the frequency $\nu_{0}$ (indicated by the Dirac $\left.\delta\left(\nu-\nu_{0}\right)\right)$, and $I^{\prime}$ is actually direction $\left(\hat{n}^{\prime}\right)$ independent. Our numerical simulation showed that it is more convenient to look at the star from the point of view of a local observer, who is static with respect to the black hole. Let this observer see the star moving with a velocity $\boldsymbol{v}=c \boldsymbol{\beta}$ with respect to his Lorentz frame. The aberration of light and the Doppler shift make him see the star having a direction $(\hat{n})$ dependent luminosity $I_{\nu}(\hat{n})$, which is distributed more heavily in the direction of motion and is radiating at bluer frequencies in this direction and redder into the opposite direction:

$$
I_{\nu}(\hat{n})=\frac{L_{0}}{4 \pi} \frac{1}{\gamma^{4}(1-\boldsymbol{\beta} \cdot \hat{n})^{3}} \delta\left(\nu-\frac{\nu_{0}}{\gamma(1-\boldsymbol{\beta} \cdot \hat{n})}\right)
$$

where

$$
\gamma=\frac{P_{0}}{\sqrt{1-\frac{2 M}{R}}}
$$

and

$$
\boldsymbol{\beta} \cdot \hat{n}=\frac{1}{A} \sqrt{1-u}\left[\sqrt{A^{2}+(1-u)\left(B-u^{2}\right)} n_{r}+u n_{\varphi}\right]
$$




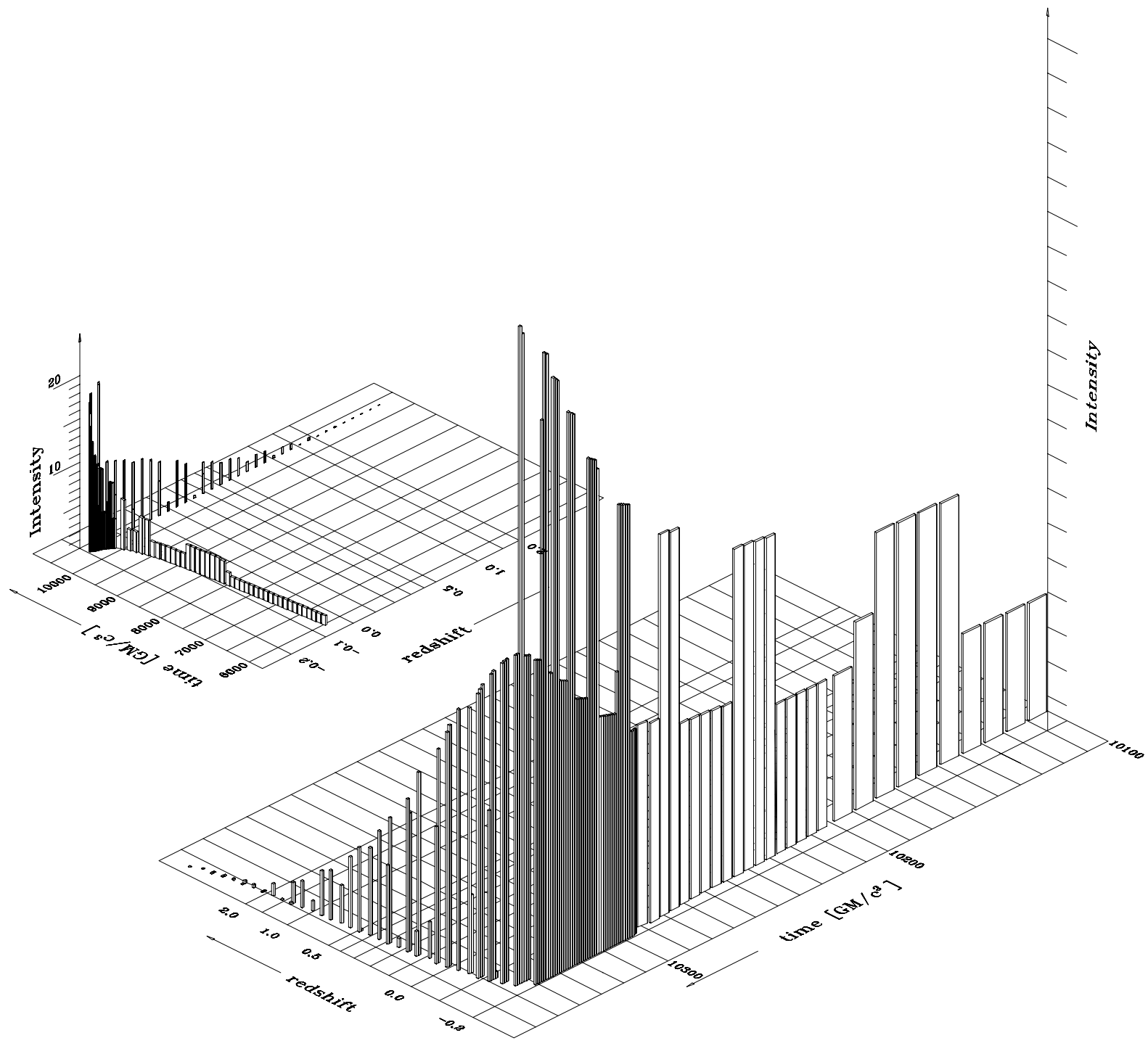

Fig. 12. The intensity and redshift as a function of time in the strip \#0. Only the last $250 G M / c^{3}$ are shown. The insert spans over the full simulation time. The curve is so rugged because the predominant contribution to light in strip \#10 comes from the thin Einstein ring around the black hole, which is (unfortunatelly) aligned with the ring pattern of emitted photons. Because of the relatively small number of photons used in the simulation, the Einstein ring is crossed by only one or two rings of photons. In other strips the gravitational lens is much less important so that they do not show the same computational defect

Here $u$ is defined by $(19), n_{r}, n_{\theta}, n_{\varphi}\left(\left(n_{r}\right)^{2}+\left(n_{\theta}\right)^{2}+\right.$ $\left.\left(n_{\varphi}\right)^{2}=1\right)$ are components of the direction vector of the emitted photon with respect to the local unit triade $\left\{\hat{e}_{r}, \hat{e}_{\theta}, \hat{e}_{\varphi}\right\}$ and $A$ and $B$ are the constants of the motion of the star defined by (21). Specific angular momentum of the photon $(1 / a)$ can be expressed with the components of the direction vector of the photon as:

$$
a=u \sqrt{\frac{1-u}{n_{\theta}^{2}+n_{\varphi}^{2}}}
$$

To calculate the illumination of the distant celestial sphere by the moving star, we divide the path of the star into equal coordinate time intervals $\left(\mathrm{d} x^{0}=\mathrm{d} t\right)$ and imagine that the star emits a burst of $N$ rays at the end of each interval. The rays $\left(\hat{n}_{i}, i=1 \ldots N\right)$ are distributed uniformly with respect to the local observer and carry the energy

$$
E_{i}=\left(\int I_{\nu}\left(\hat{n}_{i}\right) \mathrm{d} \nu\right) \frac{4 \pi}{N}\left(1-\frac{2 M}{r}\right) \mathrm{d} t
$$

and frequency

$$
\nu_{\mathrm{loc}}=\frac{\nu_{0}}{\gamma(1-\boldsymbol{\beta} \cdot \hat{n})}
$$

Each ray is propagated to the distant celestial sphere (at $r=r_{\mathrm{f}}$ ) along the lightlike geodesic tangent to $\hat{n}_{i}$ and its 


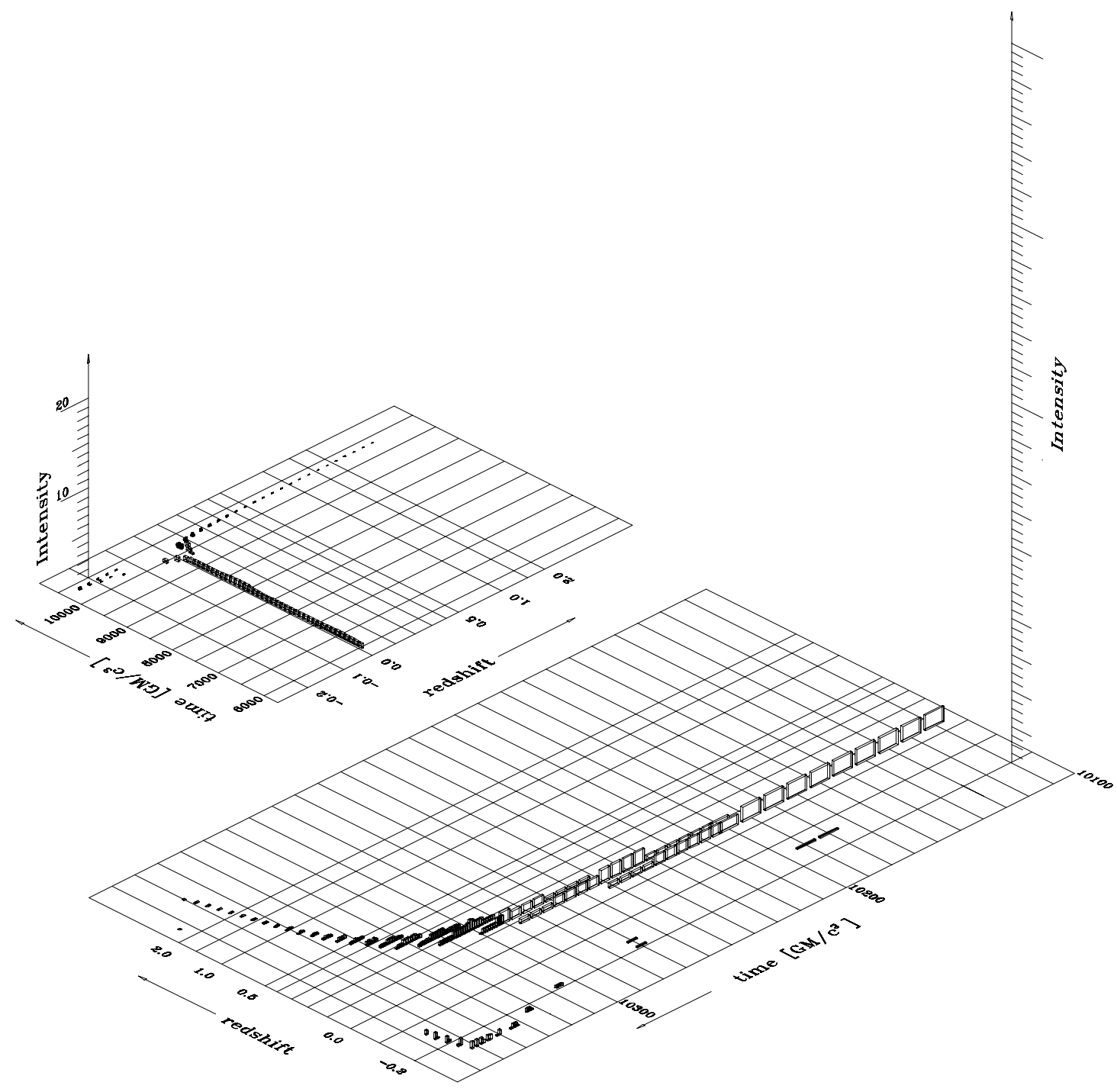

Fig. 13. Same as Fig. 12 for strip \#10. Note two families of photons reaching the far observer at later times. They have been deflected around the black hole by different paths. The blue photons have made longer trips to the observer. We expect that a simulation with a larger number of photons would show new, weaker families

final coordinates $\theta_{i}, \phi_{i}$ and its arrival time $T_{i}$ are found as described in the previous section. This ray contributes light of intensity $E_{i}$ and color, corresponding to

$$
\nu_{\infty}=\nu_{\mathrm{loc}} \sqrt{1-\frac{2 M}{r_{\mathrm{loc}}}}
$$

(the photon frequency observed at infinity) to a particular image in the sequence according to $T_{i}$.

In constructing the images as described above, it would be desirable to have at least one ray on each pixel of each image, which would require some $10^{6}$ rays per image. Considering the number of images to be calculated, this would represent too heavy a computational load for our present equipment. Therefore, we settled for a much smaller number of rays (3000) which cover the celestial sphere in a spotty fashion. In order to construct an acceptable image we blur each ray when it hits the celestial sphere so that it contributes a Gaussian spot to the image. As long as the gravitational lens effects are not very pronounced, this procedure creates a smooth image. But, when the star comes closer to the black hole, the sparseness of rays becomes responsible for some lack of detail in showing the intricacies of caustic patterns.

\section{Results}

The results of these calculations are best presented as a movie depicting the illumination of a far celestial sphere as the star is falling into a black hole. Here we present some characteristic frames of three such movies representing a 


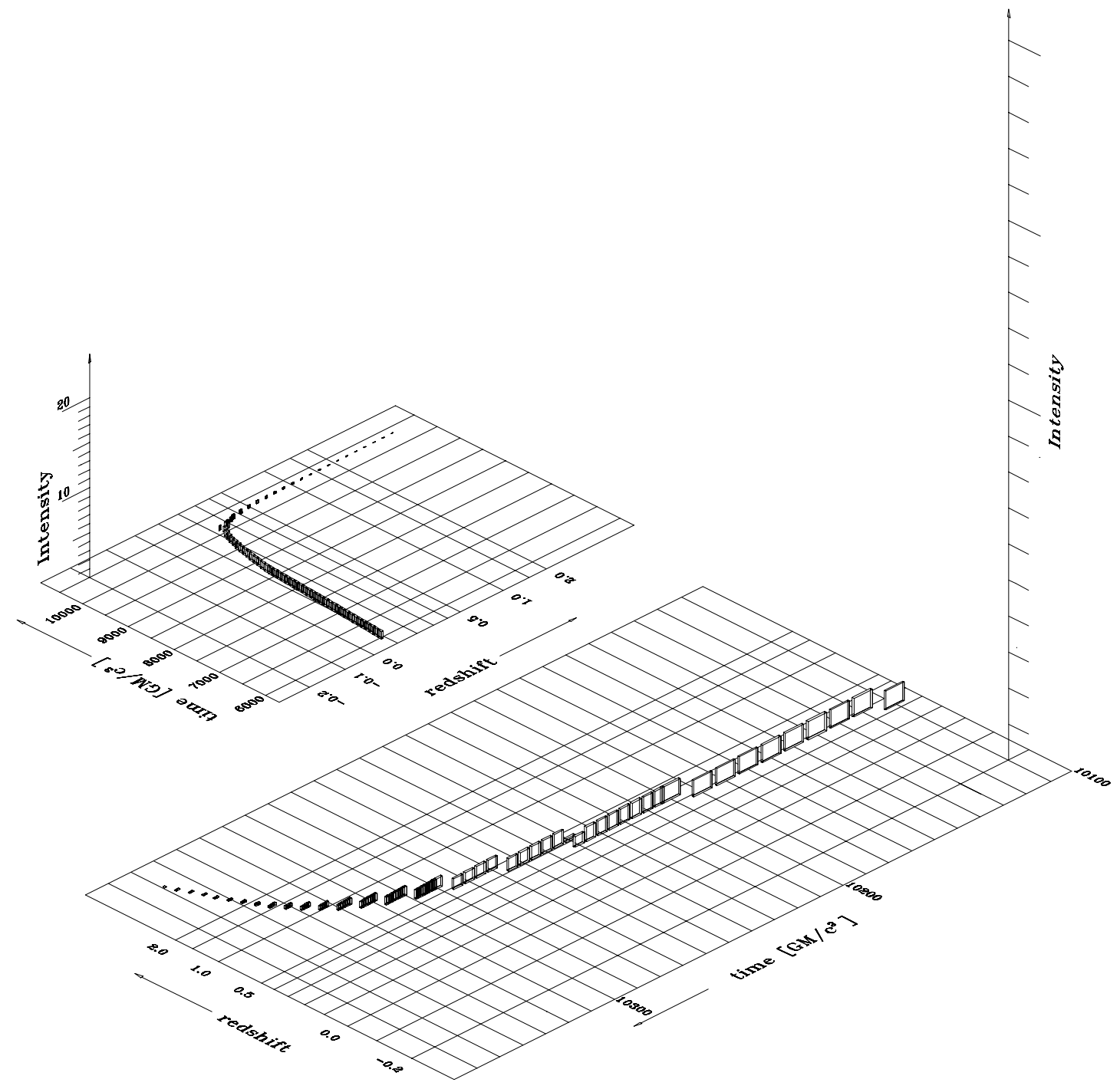

Fig. 14. Same as Fig. 12 for strip \#20. Only photons moving opposite to the velocity of the star are contributing to light on this strip. The gravitational lensing plays no significant role and the luminosity and the redshift decay monotonously. The disappearance of light occurs slightly sooner in this direction because very few photons that circle, and are thus stored around the $r=3 G M / c^{2}$ radius, contribute light to the backward direction

parabolic infall of a star into a black hole with specific angular momenta $L / M=0, L / M=2$ and $L / M=3.99$.

Figure 1 shows the coordinates used to map the celestial sphere and the color and intensity of the celestial sphere if it is illuminated by the stationary star in its centre. The color scale on top shows how different photon energies are marked.

Simulations start with $t=0$, when the star is at the distance $500 M$ from the black hole. The celestial sphere is considered to have the radius $r_{\mathrm{f}}=5000 \mathrm{M}$, so that the first light hits it at roughly $t=5000 \mathrm{M}$. Frames are calculated with the time resolution of $1 M$.

Figures 2 to 11 show some characteristic stages of radial infall for $L / M=0$. At $t=6000 M$ the star is still slow and far from the black hole, so that only moderate aberration and Doppler shift effects show up (Fig. 2). The first drastic concentration of light and blue shift in the forward direction show up at about $t=10000 M$, when the star is seen at some 30 Schwarzschild radii and is moving at $\approx 0.2 c$ (Fig. 3). As time goes on, the aberration of light and the Doppler effect continue to concentrate the intensity and the energy in the forward direction. Gravitational lensing also works in the same direction, so that the observers with respect to whom the star is approaching, see its image brightening and becoming bluer; (Figs. 4-6). The bright blue spot on the celestial sphere is more and more conspicuous until the star reaches $r=12 M$ (Fig. 6 ). At this radius the black hole is already looming in the 
sky of the star so large, that the most energetic photons emitted in the forward direction fail to reach far observers. Only light emitted at larger angles with less energy with respect to local static observers still escapes. Therefore, after $r=12 M$ is passed, all far observers start seeing less photons with lower energies. In these last moments the vicinity of the gravitational lens is the dominating agent. Since the lens focuses more and more in the forward direction, it slightly prolongs the duration of the bright spot. On the other hand, this same effect causes additional depletion of light from the backward direction, which becomes rapidly dark and red. An interesting effect is also produced by light which has been delayed by circling the black hole at the $r=3 M$ radius. This light has been emitted at small angles at, or slightly before the $r=12 \mathrm{M}$ radius has been reached, so that it has the largest blue shift. It sprinkles all over the sky for a few brief moments until it and all other light is extinguished for all observers. The described final process is very fast; in only $\approx 10 \mathrm{M}$, everything, including the bright spot on the celestial sphere reddens and fades away completely.

The interesting feature of the described process is the growth, sharpening and fast disappearance of the bright spot on the celestial sphere. In order to illustrate how this brightening might be seen by a particular observer far from the black hole, we divided the celestial sphere into 21 strips with equally spaced constant latitudes. The strip number " 0 " is the polar cap of the forward direction and the strip number 20 is its antipode. In Fig. 12 the average intensity as seen from strip \#0 is shown as a function of time and as a function of photon energies received there. Some jaggedness seen in the diagram is due to the relatively small number of photons used in the simulation. It is interesting to note that the average intensity in this strip, subtending a nonnegligible solid angle of $4 \pi \times 1.5 \cdot 10^{-3^{\mathrm{rad}}}$, has a sharp peak lasting a few $M$, which reaches almost twenty times the original brightness. The received photon energy goes up just before the sharp rise of the peak and sharply decreases with the decline of light.

The observers situated perpendicular to the motion of the star (strip \#10) see no pronounced rise in brightness. The main light component is declining and increasingly redshifting. However, shortly before everything is over, a small blue component appears and becomes dominant (Fig. 13). It corresponds to photons that have been delayed by winding around the $r=3 M$ radius, as witnessed by the slight delay of their arrival. A calculation with more photons might show light at even later times. However it is clear that its contribution can only be very weak, since the solid angle accepting photons on many-winds trajectories is very small.

Those observers seeing the star moving away (strip \#20) see its light and energy steadily declining on a time scale of roughly $100 \mathrm{M}$, which is the typical time scale on which all the described changes in the appearance of the star occur.

When the star is moving toward the black hole on a nonzero angular momentum plunging trajectory, its velocity direction is changing with time. Thus, the forward direction is not always the same and it no longer coincides with the axis of the gravitational lens. While the star is still far from the black hole, the misalignment is still small, so that the bright spot, that starts forming in the temporary forward direction on the celestial sphere, is similar to that formed in the $L=0$ case. Figures 15 17 show this development for the case $L / M=2$. As the star approaches the black hole, two things happen. The misalignment grows, and the most important contributors to the bright spot are photons with specific angular momentum $\left(l / 2 M p_{0}=1 / a\right)$ not far from the critical value $\frac{3 \sqrt{3}}{2}$. These photons suffer large gravitational bending and large time delays, so that the combined effect is difficult to guess. The simulation shows that the light continues to be focused, but with a large color gradient in the azimuthal direction. Photons contributing to the spot are of different origin and have spent different times on the way to the observer. As time goes on, and the star is approaching the $r=3 M$ radius, an increasing proportion of photons is involved in the $a=\frac{2}{3 \sqrt{3}}$ game and the pattern of the bright spot develops into intriquing caustic patterns (Fig. 18-20). Unfortunately our resolution is still not sharp enough to give the full account of the phenomenon. The maximum blue shift of 1.5 is reached by light in the bright spot for photons emitted at $r=6.69 \mathrm{M}$. The blue shift is larger in this case then in the $L=0$ case. This can be understood by the fact that the velocity of the star no longer points exactly toward the black hole and, therefore, high energy photons emitted in the forward direction can escape the black hole. After the maximum has been reached, the image of the star starts fading, becomes redder and disappears in a time interval of about $20 M$.

The star falling into the black hole on a trajectory with the angular momentum closest to the critical value $\left(L / M=4\right.$ if $\left.P_{0}=1\right)$ gives the most spectacular show before its final disappearance. In the $L / M=3.99$ case, which we have calculated, the orbit of the star makes one and a half turn before passing the horizon. The most colourful pattern is contributed by photons emitted close to $r=3 M$ for the following reason: when the star is at this radius, it is already moving very fast, predominantly in the azimuthal direction. So it is almost catching up with light emitted in the forward direction. Therefore, forward moving light emitted at different points along the trajectory reaches the far observer almost simultaneously. This effect is apparent in Figs. 20-26. As the spot becomes bluer (whiter in our color code) it is more and more focused, and the maximum blue shift reaches the factor 1.77. Soon after that - about $20 M$ later (same as before) - the spot 
and all the rest fades away and the star disappears from our sight.

If one compares the disappearance of a star behind the horizon of a black hole for different values of specific angular momentum, as seen by a particular observer, then one notes, that for $L=0$, the phenomenon looks characteristically spectacular only for observers that happen to see the star approaching. These observers will see the star brightening and becoming bluer, then dimming and becoming ever more red in a time of only some $100 \mathrm{M}$. If the star is spiralling toward the black hole, the bright spot is moving across the sky. A static observer standing close to the orbital plane of the star will see one or possibly a few light flashes with rapidly changing color and luminosity. The flashes last only about $1 M$ and repeat (if they do) in $\approx 100 M$.

\section{Conclusion}

We have presented a calculation of the appearance of an idealised star falling into a Schwarzschild black hole. The movie shows many familiar phenomena such as aberration of light, Doppler shift and gravitational lensing. However, the detailed duration and magnitude of the effect could hardly be guessed without a numerical analysis. It remains to be seen if such a phenomenon can ever be actually observed in an astrophysical event. Of course, one would not expect all the assumptions of our calculations to be fulfilled in an actual observable event. Should an ordinary star fall into a central galactic black hole, its increase in brightness would certainly be too small to be noticeable in the background luminosity of a galactic nucleus. However one expects (Carter \& Luminet 1985; Luminet \& Barbuy 1990; Luminet \& Pichon 1989a; Luminet \& Pichon 1989b) that the star will be squeezed on its way to the black hole and will most likely detonate at some stage. Such an explosion might be just what is needed to illuminate the final demise of the star.

Acknowledgements. We would like to thank Massimo Calvani for his comments and enthusiastic support while writing this article. One of us (A. Č) would also like to acknowledge the financial support of the EEC under the contract PECO 94 n.ERBCIPDCT940028 and to thank again Massimo Calvani and Fernando de Felice for their warm hospitality while part of this work was done at Osservatorio Astronomico di Padova.

\section{References}

Bardeen J.M., 1972, Black Holes. In: DeWitt C. and DeWitt B.S. (eds.), Les Houches Summer School

Carter B., Luminet J.P., 1985, MNRAS 212, 23

Gradshteyn Ryzhik, 1965, Tables of Integrals, Series and Products. Academic Press, San Diego

Gurzadyan V.G., Ozernoy L.M., 1981, A\&A 95, 39

Luminet J.P., Barbuy B., 1990, AJ 99, 838-842

Luminet J.P., Carter B., 1986, ApJS 61, 219

Luminet J.P., Marck J.A., 1985, MNRAS 212, 57

Luminet J.P., Pichon B., 1989a, A\&A 209, 85

Luminet J.P., Pichon B., 1989b, A\&A 209, 103

Misner C.W., Thorne K.S., Wheeler J.A., 1973, Gravitation. In: Freeman W.H. and Co. (eds.), San Francisco

Piran T., 1995, Sci. Am. 272, 34

Rauch K.P., Blandford R.D., 1994, ApJ 421, 46

Rees M.J., 1988, Nat 333, 523

Rees M.J., 1990, Sci 247, 817

INTERNET: "CGRO/OSSE NU Gamma Ray Burst Index"

Weinberg S., 1972, Gravitation and Cosmology. J. Wiley and Sons, New York 